\title{
AMBIÊNCIA AÉREA EM MATERNIDADE E CRECHE DE SUÍNOS ${ }^{1}$
}

\section{NEIDIMILA A. SILVEIRA ${ }^{2}$, IRENILZA DE A. NÄÄS ${ }^{3}$, DANIELLA J. DE MOURA ${ }^{4}$, DOUGLAS D'ALESSANDRO SALGADO ${ }^{5}$}

RESUMO: A suinocultura brasileira é uma atividade importante para o agronegócio do País. Embora o uso de tecnologia nos vários segmentos dessa atividade envolva a substituição do trabalho humano pela automatização de tarefas, o uso da mão de obra humana ainda é significativo. O trabalhador no setor suinícola está exposto a uma série de fatores de risco na execução de suas atividades, dentre os quais a exposição a gases nocivos. $\mathrm{O}$ presente trabalho teve por objetivo avaliar a qualidade do ar em instalações de maternidade e creche suinícolas, em duas granjas, com diferentes características (G1 e G2). Para tanto, foram avaliadas concentração de gases $\left(\mathrm{NH}_{3}, \mathrm{CO}_{2} \mathrm{e}\right.$ $\mathrm{CO})$ e poeira respirável durante o período de atividades dos trabalhadores. As concentrações dos gases avaliados estiveram dentro dos limites recomendados pela NR-15 (1978) e ACGIH (2001). O gás que apresentou maior concentração foi $\mathrm{NH}_{3}$ no galpão de creche, que atingiu a concentração de $10 \mathrm{ppm}$, não ultrapassando os limites recomendados. As concentrações de poeira respirável mantiveram-se dentro dos limites estabelecidos pelas normas ACGIH (2001), NR-15 (1978) e NIOSH (1994), não havendo diferença significativa entre os galpões avaliados.

PALAVRAS-CHAVE: suinocultura, ambiente aéreo, trabalhador.

\section{AERIAL ENVIRONMENT IN FARROWING AND SWINE NURSERY}

ABSTRACT: Brazilian swine production is an important activity for the agribusiness, and even though the use of technology in various segments of this activity allowed mechanization of processes, intensive labor is still needed. Swine worker is exposed to several risk factors during their activities, among them the exposition to noxious gases. The present research had as general objective to evaluate gases concentration $\left(\mathrm{NH}_{3}, \mathrm{CO}, \mathrm{CO}_{2}\right)$ and wind speed during the workers activities. It was found that the gases concentration $\left(\mathrm{NH}_{3}, \mathrm{CO}, \mathrm{CO}_{2}\right)$ are within the limits recommended by NR15 (1978) and CGIH (2001). Gases concentration values found were low besides ammonia concentration found in G1 farms inside the nursing housing during the morning $(10 \mathrm{ppm})$ that is within the recommended value. Inhalable dust concentration values found were within the limits established by the norms ACGIH (2001), NR-15 (1978) and NIOSH (1994) in both houses, and there was not statistical significance between them.

KEYWORDS: swine production, air environment, worker.

\footnotetext{
${ }^{1}$ Extraído da dissertação de mestrado da primeira autora.

${ }^{2}$ Fisioterapeuta, Mestre em Engenharia Agrícola, Faculdade de Engenharia Agrícola, UNICAMP, Cidade Universitária Zeferino Vaz, s/n, Campinas - SP, Fone: (0XX19) 3521.1129, neidimila@gmail.com

${ }^{3}$ Profa. Colaboradora, Faculdade de Engenharia Agrícola, UNICAMP, Campinas - SP, irenilza@ agr.unicamp.br

${ }^{4}$ Profa. Assistente Doutora, Faculdade de Engenharia Agrícola, UNICAMP, Campinas - SP.

${ }^{5}$ Estatístico, Doutorando, Faculdade de Engenharia Agrícola, UNICAMP, Campinas - SP.

Recebido pelo Conselho Editorial em: 2-1-2008

Aprovado pelo Conselho Editorial em: 24-8-2009
} 


\section{INTRODUÇÃO}

A partir da década de 1960, as antigas criações extensivas de suínos passaram a se intensificar, caracterizando-se pelo alojamento de grande número de animais em espaço físico reduzido. Isso tornou possível o grande aumento na produção de alimentos de origem animal para consumo humano (BECKER, 2004).

No Brasil, a carne suína é a terceira mais importante carne em volume de produção, sendo superada pela produção das carnes bovina e de aves. Essa atividade é uma das mais importantes para a formação do Produto Interno Bruto brasileiro, e constitui extraordinário gerador de empregos (ANUALPEC, 2004).

Os suínos liberam diretamente para o ar: calor, umidade, dióxido de carbono proveniente do consumo de oxigênio, gases da digestão e poeira. Indiretamente, outros produtos são liberados para o ar oriundos dos excrementos, tais como: calor, umidade, gases da digestão aeróbia e anaeróbia de dejetos (carbônico, amônia e sulfídrico) e poeira liberada do piso e ração (VERSTEGEN et al.,1994; PIFFER et al., 1998; HANNAS, 1999; PERDOMO et al., 2001; NADER et al., 2002).

Os sintomas respiratórios nos trabalhadores rurais estão, em geral, associados às atividades realizadas em silos e no confinamento animal, e principalmente correlacionadas à poeira existente nesses ambientes de trabalho. Problemas respiratórios surgem (asthma-like syndrome, farmer's lung, e silo filler's disease) pela exposição à poeira, bem como à inalação de esporos de fungos (MUTHEL \& DONHAM, 1983). O ambiente de trabalho deve apresentar-se saudável ao trabalhador. Nos galpões de produção de suínos, existe risco evidente relacionado aos aspectos químicos e microbiológicos, decorrentes do ambiente de alojamento de suínos. As legislações brasileira e internacional (NR-15, 1978; OSHA, 1994; ACGIH, 2001) estabelecem limites de exposição para os tipos de poeira mineral e orgânica, para ambas as frações inalável e respirável.

A preocupação em adequar a ferramenta ao ser humano mostrou-se mais evidente após a $2^{\underline{a}}$ Grande Guerra, quando os avanços da ciência e da tecnologia foram sendo aplicados às características e limitações do homem, com o intuito de alcançar melhor desempenho, redução da fadiga e dos acidentes (SALIBA, 1997; MÁSCIA \& SZNELWAR, 2001).

O objetivo do presente trabalho foi avaliar a qualidade do ar em instalações de maternidade e creche para diferentes unidades de produção suinícola, por meio do registro da concentração de gases: amônia $\left(\mathrm{NH}_{3}\right)$, dióxido de carbono $\left(\mathrm{CO}_{2}\right)$, monóxido de carbono $(\mathrm{CO})$, e poeira respirável, quanto à exposição do trabalhador.

\section{MATERIAL E MÉTODOS}

Esta pesquisa foi realizada em duas granjas (G1 e G2) de produção suinícolas nos setores de creche e maternidade. Foram identificadas as tarefas dos trabalhadores: corte de cauda, corte de umbigo, trato, manejos no parto, lixar dente, limpeza do galpão, nas etapas de produção maternidade e creche, por serem as etapas em que se concentra o maior número de tarefas.

Os dados foram registrados nos períodos da manhã e da tarde, sendo três coletas para cada granja. As coletas foram realizadas durante algum manejo, mas não houve interferência no manejo empregado, a 1,5 m do piso, sendo essa distância recomendada para a avaliação voltada ao trabalhador. Os equipamentos foram alocados no centro geométrico das salas avaliadas. Na Tabela 1, apresentam-se as características tipológicas e os espaçamentos das instalações estudadas. 
TABELA 1. Tipologia das instalações de maternidade e de creche de suínos para as duas granjas utilizadas no experimento. Typology of the facilities of swine nursery and farrowing for two farms used in the experiment.

\begin{tabular}{|c|c|c|c|c|}
\hline \multirow{2}{*}{$\begin{array}{l}\text { Características da } \\
\text { Construção }\end{array}$} & \multicolumn{2}{|c|}{ Granja 1} & \multicolumn{2}{|c|}{ Granja 2} \\
\hline & Creche & Maternidade & Creche & Maternidade \\
\hline Área da sala $\left(\mathrm{m}^{2}\right)$ & 173 & 279 & 200 & 72 \\
\hline Material de construção & alvenaria & alvenaria & alvenaria & alvenaria \\
\hline Tipo de piso & cimentado & $\begin{array}{l}\text { semi-ripado e } \\
\text { cimentado }\end{array}$ & $\begin{array}{l}\text { semi-ripado e } \\
\text { cimentado }\end{array}$ & $\begin{array}{l}\text { semi-ripado e } \\
\text { cimentado }\end{array}$ \\
\hline Tipo de confinamento & Baias & gaiolas & baias suspensas & gaiolas \\
\hline Orientação do galpão & leste-oeste & leste-oeste & leste-oeste & norte-sul \\
\hline Pé-direito (m) & 2,20 & 3,26 & 2,23 & 2,30 \\
\hline Material de telhado & fibrocimento & cerâmica & cerâmica & cerâmica \\
\hline Forro & utiliza & não utiliza & não utiliza & não utiliza \\
\hline Uso ou não de cama & não utiliza & não utiliza & utiliza & não utiliza \\
\hline Volume do ambiente $\left(\mathrm{m}^{2}\right)$ & 380 & 909 & 446 & 165 \\
\hline $\begin{array}{l}\text { Peso total de animal }(\mathrm{kg}) / \\
\text { volume de ambiente }\left(\mathrm{m}^{3}\right)\end{array}$ & 16,81 & 7,91 & 7,84 & 9,66 \\
\hline $\begin{array}{l}\text { Relação área de janela / área } \\
\text { de piso }\end{array}$ & 0,13 & 0,32 & 0,15 & 0,13 \\
\hline
\end{tabular}

\section{Granja 1 (G1)}

Localizada na região de Salto - SP, à latitude de $23^{\circ} 12^{\prime} 10^{\prime \prime} \mathrm{S}$, longitude $47^{\circ} 17^{\prime} 35^{\prime}$ ' W e altitude de $521 \mathrm{~m}$, e clima predominante Cwa (classificação de Köeppen), caracterizado como clima quente, temperado chuvoso, com estação seca no inverno e com verão quente. A granja possuia plantel médio de 930 matrizes de genética própria formada por fêmea (Landrace x Large White) e macho (Pic) e contava com 30 funcionários.

\section{Creche 1}

Instalação com pilares de concreto armado, telhado em duas águas com lanternim $(1,90 \mathrm{~m}$ de abertura horizontal) e o entorno das instalações de gramado. A edificação era formada por quatro salas separadas por paredes de $1 / 2$ tijolo, sendo cada sala formada por duas baias separadas por parede (1/2 tijolo) na altura de $1,0 \mathrm{~m}$. Cada sala possuía média de 400 suínos que variavam de 40 a 55 dias de vida.

\section{Maternidade 1}

Instalação com pilares de concreto armado e o entorno formado por grama de um lado e cimento de outro. A edificação era formada por três salas separadas. Cada sala possuía área de piso cimentado de $279,12 \mathrm{~m}^{2}(23,26 \mathrm{~m} \times 12,00 \mathrm{~m})$ e os corredores variavam de $0,61 \mathrm{~m}$ a $1,16 \mathrm{~m}$ de largura. Os fechamentos laterais eram de alvenaria ( $1 / 2$ tijolo) na altura de $1,50 \mathrm{~m}$, com cortina para controle da ventilação natural. As baias (gaiolas) possuíam área de piso de 4,24 $\mathrm{m}^{2}(2,70$ x 1,57), contando com a área do escamoteador. Possuíam mureta de 0,61 m de altura. Cada sala possuía 36 gaiolas, sendo seis gaiolas por corredor. Cada matriz tinha média de 10 leitões.

\section{Granja 2 (G2)}

As instalações localizam-se na região de Bragança Paulista - SP, situada à latitude de $22^{\circ} 57^{\prime} 07^{\prime}$ ' S, longitude $46^{\circ} 32^{\prime} 31^{\prime}$ ' W, e altitude média de $817 \mathrm{~m}$. O clima predominante da região é Cwa (classificação de Köeppen), caracterizado como clima tropical de altitude. A granja possuía plantel médio de 280 matrizes de genética mista: Landrace, Large White e Pic, num total de 2.400 animais, com 10 funcionários. 


\section{Creche 2}

Instalação com pilares de concreto armado e o entorno cercado por grama e terra batida. A sala era formada por 16 baias separadas por parede ( $1 / 2$ tijolo), sendo oito baias de cada lado do corredor de 1,0 m de largura no centro, entre as baias, para os serviços de rotina. As baias possuíam área de piso de 4,08 $\mathrm{m}^{2}$ (1,40 m x 2,92 m), sendo semirripado e parte cimentada. $O$ galpão utilizado para as coletas de dados possuía 250 suínos, com peso médio de $10 \mathrm{~kg}$ (35 dias de vida, aproximadamente).

\section{Maternidade 2}

Instalação com pilares de concreto armado e telhado em duas águas, com lanternim. $\mathrm{O}$ entorno da instalação era coberto por grama. $\mathrm{O}$ galpão utilizado neste estudo possuía cinco salas; cada sala apresentava área de piso de $72 \mathrm{~m}^{2}(8,0 \times 9,0 \mathrm{~m})$ e, em cada uma delas, havia oito matrizes, sendo uma em cada gaiola, com 11 leitões, em média.

\section{Medida de gases}

Para a avaliação da concentração de $\mathrm{NH}_{3}, \mathrm{CO}_{2}$ e $\mathrm{CO}$, foram coletadas amostras de ar instantâneas, utilizando bomba de sucção e tubetes contendo reagentes, da marca Dräger ${ }^{\circledR}$. Para a detecção de $\mathrm{CO}_{2}$, foi usado o tubo reagente a partir de amostra de $100 \mathrm{~cm}^{3}$, com intervalo de 100 a 3.000 ppm. Para a detecção de $\mathrm{NH}_{3}$, foi utilizado o tubo reagente a partir de amostra de $2 \mathrm{~cm}^{3}$, com intervalo de 2 a 30 ppm, e para a detecção de CO, foi usado o tubo reagente a partir de amostra de $100 \mathrm{~cm}^{3}$, com intervalo de 2 a $60 \mathrm{ppm}$. A amostra de ar de $100 \mathrm{~cm}^{3}$ era succionada para dentro da bomba, passando pelo tubo reagente acoplado a sua extremidade, que continha reagente em presença de cristal, sendo a concentração do gás avaliada por meio da leitura da escala graduada situada na parede do tubo.

\section{Medida de poeira}

A avaliação de poeira foi realizada com amostras de $1 \mathrm{~h}$, no período da manhã, e $1 \mathrm{~h}$, no período da tarde, em cada galpão. Para a amostragem de poeira, foi utilizada bomba de uso individual, alimentada por baterias de níquel/cádmio recarregáveis, com capacidade de vazão de 1 a $5 \mathrm{~L} \min ^{-1}$ (Modelo Gilair-5 - Gilian ${ }^{\circledR}$ ), que aspira o ar contaminado até o filtro. Para a pesagem do filtro, antes e após a coleta de poeira, foi utilizada balança analítica eletrônica (Interprise/ADAM Equipment CO.), com capacidade de $210 \mathrm{~g}$ e sensibilidade de $0,001 \mathrm{~g}$. A avaliação de poeira respirável foi realizada de acordo com os métodos padronizados (NIOSH, 1994).

Este foi um estudo observacional de caráter comparativo, para duas granjas (G1 e G2), e, para tanto, foi realizada análise comparativa utilizando gráficos de BoxPlot, gráficos de intervalo de confiança e Teste de Kruskal Wallis, com 5\% de significância, por se tratar de dados não paramétricos, utilizando o programa computacional estatístico MINITAB-14 ${ }^{\circledR}$.

\section{RESULTADOS E DISCUSSÃO}

Os resultados de concentração dos gases $\mathrm{CO}, \mathrm{CO}_{2}$ e $\mathrm{NH}_{3}$ encontram-se dentro dos limites recomendados pela NR-15 (1978), nos galpões de creche e maternidade nas granjas G1 e G2 (Tabela 2). Nota-se, na FIGURA1, que os valores de $\mathrm{NH}_{3}$ não ultrapassaram os limites estabelecidos pela ACGIH (2001) e NR-15 (1978). O maior valor encontrado foi na G1, no galpão creche, no período da manhã, onde atingiu $10 \mathrm{ppm}$. A G1, de modo geral, apresentou maior dispersão de dados, comparada à $\mathrm{G} 2$, contudo os níveis de amônia $\left(\mathrm{NH}_{3}\right)$, para ambas as granjas, são similares, não havendo diferença significativa, de acordo com o teste de Kruskal-Wallis $(\alpha=$ 0,05). DONHAM \& CUMRO (1999) recomendam o limite máximo de 7 ppm para os trabalhadores rurais em geral, considerando a possibilidade de aparecimento de sintomas respiratórios agudos e crônicos quando da exposição a esses valores. Acima desse valor, os autores observaram perdas na capacidade respiratória desses trabalhadores, desconsiderando o fator tempo. Já BARKER et al. (2002) descrevem que, na exposição entre 6 a 20 ppm de $\mathrm{NH}_{3}$ e acima, verifica-se irritação nos olhos e problemas respiratórios. Portanto, o resultado da creche da G1, no período da manhã, registrou ponto crítico de concentração de amônia. 
TABELA 2. Concentrações de $\mathrm{CO}, \mathrm{CO}_{2}$ e $\mathrm{NH}_{3}$ nos setores de creche e maternidade das granjas $\mathrm{G} 1$ e G2. Concentrations of $\mathbf{C O}, \mathrm{CO}_{2}$ and $\mathrm{NH}_{3}$ in the sectors of farrowing and swine nursery in G1 and G2 farms.

\begin{tabular}{|c|c|c|c|c|c|c|}
\hline Galpão & Granja & Dia & Período & $\mathrm{CO}_{2}(\mathrm{ppm})$ & $\mathrm{CO}(\mathrm{ppm})$ & $\mathrm{NH}_{3}(\mathrm{ppm})$ \\
\hline \multirow{12}{*}{ Creche } & \multirow{6}{*}{ G1 } & \multirow{2}{*}{1} & manhã & 700 & $<2$ & 10,00 \\
\hline & & & tarde & 750 & $<2$ & 4,00 \\
\hline & & \multirow{2}{*}{2} & manhã & 1.000 & $<2$ & 1,80 \\
\hline & & & tarde & 1.500 & $<2$ & 2,00 \\
\hline & & \multirow{2}{*}{3} & manhã & 600 & $<2$ & 1,00 \\
\hline & & & tarde & 600 & $<2$ & 1,00 \\
\hline & \multirow{6}{*}{$\mathrm{G} 2$} & \multirow{2}{*}{1} & manhã & 550 & $<2$ & 1,00 \\
\hline & & & tarde & $*$ & $*$ & $*$ \\
\hline & & \multirow{2}{*}{2} & manhã & 450 & $<2$ & 1,00 \\
\hline & & & tarde & * & $*$ & $*$ \\
\hline & & \multirow{2}{*}{3} & manhã & 450 & $<2$ & 1,00 \\
\hline & & & tarde & 450 & $<2$ & 1,00 \\
\hline \multirow{12}{*}{ Maternidade } & \multirow{6}{*}{ G1 } & \multirow{2}{*}{1} & manhã & 550 & $<2$ & 4,00 \\
\hline & & & manhã & $*$ & $*$ & $*$ \\
\hline & & \multirow{2}{*}{2} & manhã & 400 & $<2$ & 2,00 \\
\hline & & & tarde & 150 & $<2$ & 1,80 \\
\hline & & \multirow{2}{*}{3} & manhã & 600 & $<2$ & 2,00 \\
\hline & & & tarde & 850 & $<2$ & 3,50 \\
\hline & \multirow{6}{*}{ G2 } & \multirow{2}{*}{1} & manhã & 600 & $<2$ & 4,00 \\
\hline & & & tarde & 550 & $<2$ & 3,00 \\
\hline & & \multirow{2}{*}{2} & manhã & 650 & $<2$ & 3,00 \\
\hline & & & tarde & 500 & $<2$ & 3,00 \\
\hline & & \multirow{2}{*}{3} & manhã & 600 & $<2$ & 5,00 \\
\hline & & & tarde & 450 & $<2$ & 2,50 \\
\hline
\end{tabular}

*Não houve coleta

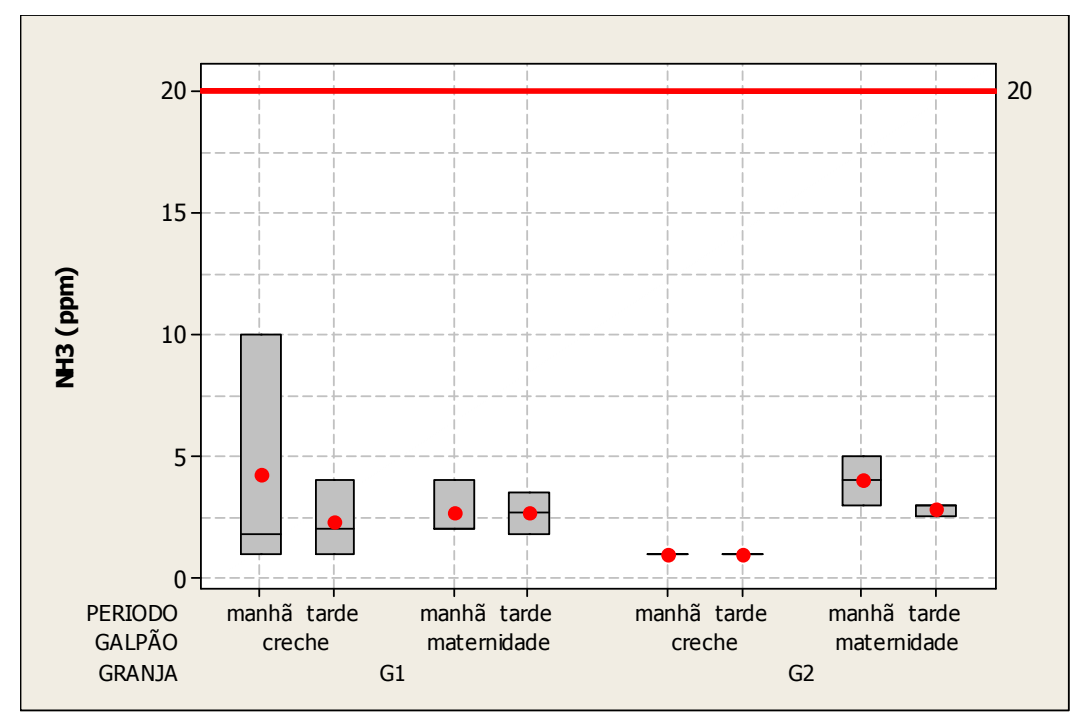

FIGURA 1. Comparação dos níveis de concentração de $\mathrm{NH}_{3}$, nos setores de creche e de maternidade de suínos, nos períodos da manhã e da tarde, nas granjas G1 e G2. Comparison of the concentration level of $\mathrm{NH}_{3}$ in the sectors of farrowing and swine nursery in morning and afternoon periods in G1 and G2 farms. 
$\mathrm{O}$ intervalo de confiança da média, para os dados do gás $\mathrm{NH}_{3}$, nas granjas G1 e G2, nos galpões de creche e de maternidade, nos períodos da manhã e da tarde, estão apresentados na Figura 2. Destacam-se os pontos médios com seus respectivos intervalos de confiança de 95\%, demonstrando que não houve diferença entre as médias. Isso significa que, embora os galpões e as respectivas salas de creche e de maternidade tivessem diferenças tipológicas, essas não influenciaram na concentração de amônia resultante. Nota-se, também na Figura 2, que houve dispersão maior dos valores registrados nas salas de creche e de maternidade da G1, indicando que a relação peso - vivo por volume da sala na creche $(16,81)$, associado ao fato de ter forro e relação área de janela com área de piso menor $(0,13)$ do que a G2 $(0,15)$, pode ter contribuído para esse resultado.

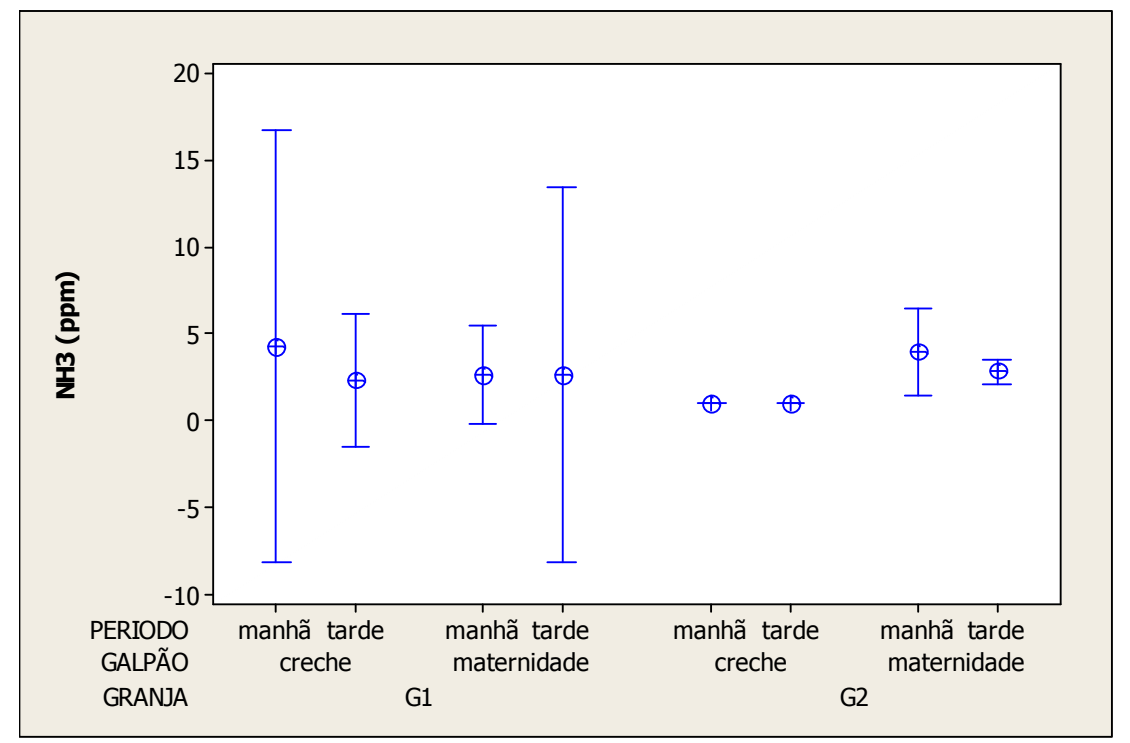

FIGURA 2. Intervalo de confiança da média de concentração de $\mathrm{NH}_{3}$, nas granjas $\mathrm{G} 1$ e $\mathrm{G} 2$, nos galpões de creche e de maternidade de suínos, nos períodos da manhã e da tarde. Confidence interval of the mean concentration of $\mathrm{NH}_{3}$ in $\mathrm{G1}$ and $\mathrm{G} 2$ farms in the farrowing and swine nursery houses in morning and afternoon periods.

Os valores de $\mathrm{CO}$ mantiveram-se constantes em G1 e G2 e nos dois galpões, situando-se dentro dos limites de salubridade. $\mathrm{O}$ valor encontrado foi sempre inferior a $2 \mathrm{ppm}$, estando em conformidade com estudos anteriores (SAMPAIO, 2004; NI et al., 2002; GUSTAFSSON, 1997; PICKRELL, 1991; BENEDI, 1986).

As concentrações de $\mathrm{CO}_{2}$, em G1 e G2, nas salas de creche e de maternidade, nos períodos da manhã e da tarde, são similares (Figura 3), não havendo diferença significativa, de acordo com o teste de Kruskal-Wallis $(\alpha=0,05)$. Porém, todos os valores encontrados em G1, nas salas de creche e de maternidade, foram numericamente superiores aos de G2. Aqui novamente a ventilação de G1 pode ser deficiente para a dispersão dos gases. $\mathrm{Na}$ G1, na sala de creche, os valores de $\mathrm{CO}_{2}$ alcançaram $1.500 \mathrm{ppm}$, no período da tarde. LARRY et al. (1994) afirmam que o dióxido de carbono $\left(\mathrm{CO}_{2}\right)$ é excessivo acima de 3.000 ppm e tem origem na respiração dos animais, sendo sua presença indicativa da qualidade do ar e da eficiência da ventilação como alternativa para diminuir sua concentração. Segundo ACGIH (2001), o $\mathrm{CO}_{2}$ tem o limite de exposição ocupacional de 5.000 ppm, enquanto a NR-15 (1978) coloca como sendo de 3.900 ppm o limite máximo, portanto os resultados indicam que os trabalhadores se encontram em condições de salubridade. 


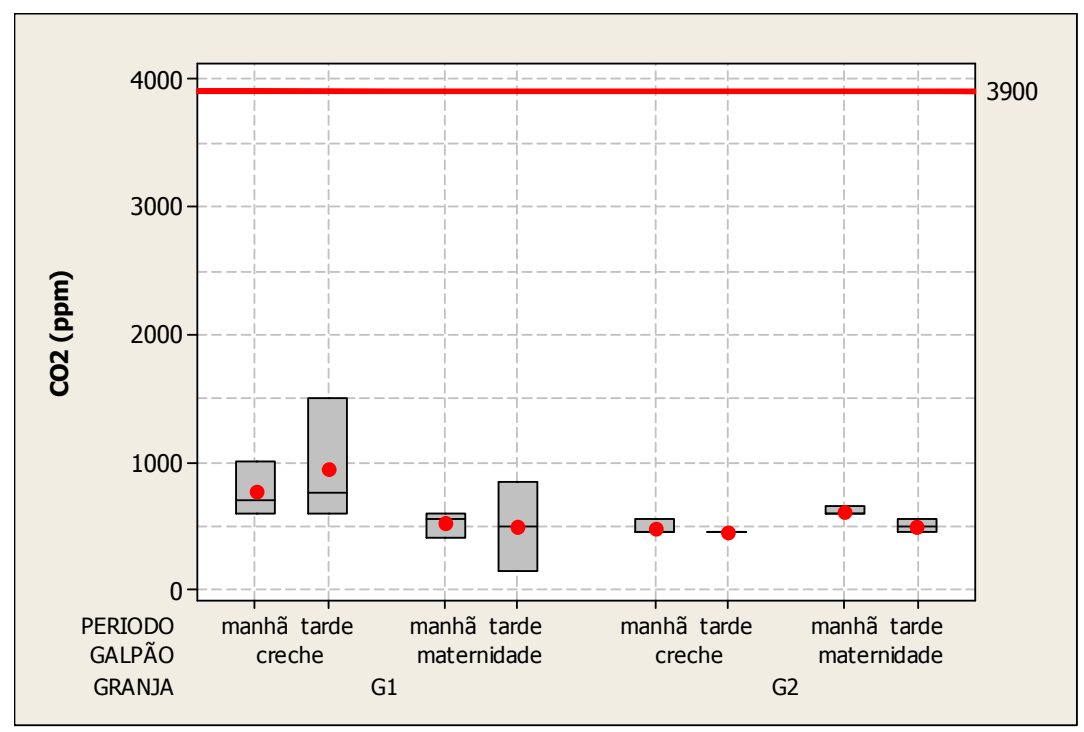

FIGURA 3. Comparação dos níveis de concentração de $\mathrm{CO}_{2}$ entre as granjas G1 e G2, nos galpões de creche e maternidade de suínos, nos períodos da manhã e da tarde. Comparison of concentration level of $\mathrm{CO}_{2}$ between $\mathrm{G} 1$ and $\mathrm{G} 2$ farms in the farrowing and swine nursery houses, in morning and afternoon periods.

Não houve diferença entre as médias, com intervalo de confiança de $95 \%$, para os dados do gás $\mathrm{CO}_{2}$, nas granjas $\mathrm{G} 1$ e $\mathrm{G} 2$, nas respectivas salas de creche e de maternidade, nos períodos da manhã e da tarde (Figura 4).

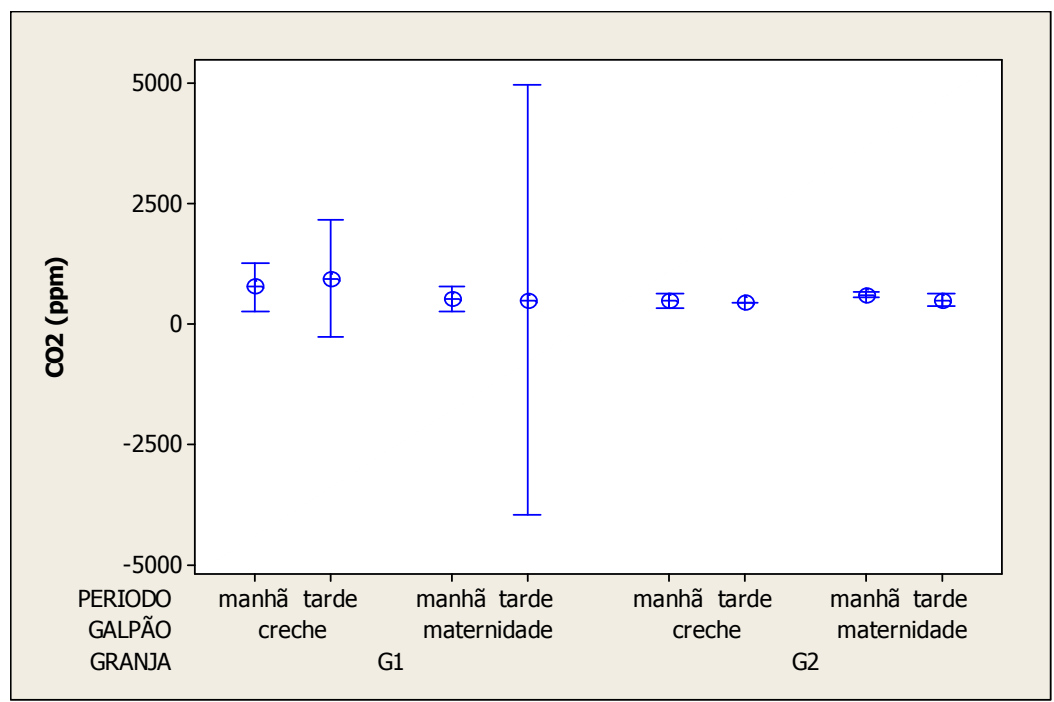

FIGURA 4. Intervalo de confiança da média de concentração de $\mathrm{CO}_{2}$ entre granjas $\mathrm{G} 1$ e $\mathrm{G} 2$, nos galpões de creche e de maternidade de suínos, nos períodos da manhã e da tarde. Confidence interval of the mean concentration of $\mathrm{CO}_{2}$ between $\mathrm{G} 1$ and $\mathrm{G} 2$ farms in farrowing and swine nursery houses, in morning and afternoon periods.

As concentrações de poeira respirável mantiveram-se dentro dos limites estabelecidos pelas normas ACGIH (2001), NR-15 ou 1978 e NIOSH (1994), nas salas de creche e de maternidade, em ambas G1 e G2 (Tabela 3), concordando com os resultados obtidos por PICKRELL (1991). 
TABELA 3. Concentração de poeira respirável nas salas de creche e de maternidade, em ambas as granjas estudadas. Concentration of inhalable dust in the rooms of farrowing and swine nursery, in both studied farms.

\begin{tabular}{cccc}
\hline & \multicolumn{3}{c}{ Poeira Respirável $\left(\mathrm{mg} \mathrm{m}^{-3}\right)$} \\
\hline \multirow{2}{*}{ Granja 1 } & Galpão & Manhã & Tarde \\
\cline { 2 - 4 } & Creche & 2,9 & 1,7 \\
\multirow{2}{*}{ Granja 2 } & Maternidade & 1,9 & 0,5 \\
& Creche & 2,0 & 2,2 \\
& Maternidade & 0,5 & 0,2 \\
\hline
\end{tabular}

As concentrações de poeira foram mais altas nos galpões de creche, em comparação com os galpões de maternidade (Tabela 3). Resultado que também foi relatado por outros pesquisadores, que estudaram condições de ambiente aéreo em suinocultura (SAMPAIO et al., 2007; CHANG et al., 2001; GUINGAND, 1999). Esses valores podem ser justificados pela densidade populacional e pelas características construtivas do galpão, pois os galpões de creche são mais fechados e com pouca circulação de ar, também pelo tipo de manejo e pela atividade dos animais.

Embora dentro dos limites, se indivíduos apresentarem hipersensibilidade a esses agentes, o ambiente pode ser interpretado como insalubre, conforme afirmam ALENCAR et al. (2002). Por outro lado, indivíduos normais expostos constantemente a poluentes podem apresentar alterações no sistema imunológico, com redução do "clearance" mucociliar (MARTINS et al., 2002), sendo, portanto, a questão do tempo de exposição uma condição importante a ser considerada.

\section{CONCLUSÕES}

As concentrações dos gases $\mathrm{CO}, \mathrm{CO}_{2}$ e $\mathrm{NH}_{3}$ estão dentro dos limites recomendados pela NR-15 (1978) e ACGIH (2001) para os setores e granjas estudadas.

As concentrações de poeira respirável mantiveram-se dentro dos limites estabelecidos pelas normas ACGIH (2001), NR-15 (1978) e NIOSH (1981), e foram mais altas nas salas de creche, em comparação com as salas de maternidade.

\section{AGRADECIMENTOS}

À FAPESP e ao CNPq, pelo apoio financeiro à pesquisa, e à CAPES, pela bolsa concedida.

\section{REFERÊNCIAS}

\section{ACGIH. AMERICAN CONFERENCE OF GOVERNMENT INDUSTRIAL HYGIENISTS.} Cincinnati. TLVS and BEIs - Threshold Limit Values for Chemical Substances and Biological Exposure Indices, Cincinnati, 2001. 185p.

ALENCAR, M.C.B.; GONTIJO, L.A.; NÄÄS, I.A.; BARACHO, M.S.; MIRAGLIOTTA, M.Y. A saúde ocupacional na produção de frangos de corte no Brasil: será que sabemos o bastante? In: SEMINÁRIO POLUENTES AÉREOS E RUÍDOS EM INSTALAÇÕES PARA PRODUÇÃO DE ANIMAIS, 2002, Campinas. Anais... p.57-62. 1 CD-ROM.

BARKER, J.; CURTIS, S.; HOGSETT, O.; HUMENIK, F. Safety in swine productions systems. North Carolina: Waste Quality \& Waste Management, North Carolina Cooperative Extension Service, 2002. $12 \mathrm{p}$.

ANUALPEC 2004: Anuário da Pecuária Brasileira. São Paulo: FNP Consultoria e Comércio, 2004. p.267-299.

BECKER, G.B. Bem-estar animal. Disponível em: <http // www. Porkworld.com.br>. Acesso em: 9 fev. 2004. 
BENEDI, J.M.H. El ambiente de los alojamentos ganaderos. Hojas Divulgadoras, Madrid, n.6, p.68-28, 1986.

CHANG, C.W.; CHUNH, H.; HUANG, C.F.; SU, H.J.J. Exposure assessment to airbone endotoxin, dust, ammonia, hydrogen sulfide and carbon dioxide in open style swine houses. Annals of Occupational Hygene, New York, v.45, n.6, p.457-465, 2001.

DONHAM, K.; CUMRO, D. Setting maximum dust exposure levels for people and animal in livestock facilities. In: INTERNATIONAL SYMPOSIUM ON DUST CONTROL IN ANIMAL PRODUCTION FACILITIES, 1., 1999, Aarhus, Denmark. Proceedings...Horsens, Denmark: Danish Institute of Agricultural Sciences, 1999. 1 CD-ROM.

GUINGAND, N. Dust concentrations in piggeries: influence of season, age of pigs, type of floor and feed presentation in farrowing, post-weaning and finishing rooms. In: INTERNATIONAL SYMPOSIUM ON DUST CONTROL IN ANIMAL PRODUCTION FACILITIES, 30., 1999, Aarhus, Denmark. Proceedings... Horsens, Denmark: Danish Institute of Agricultural Sciences, 1999. p.69-75.

GUSTAFSSON, B. The health and safety of workers in a confined animal system. Livestock Production Science, Amsterdan, v.49, n.1, p. 123-136, 1997.

HANNAS, M.I. Aspectos fisiológicos e a produção de suínos em clima quente. In: SILVA, I.J.O. Ambiência e qualidade na produção industrial de suínos. Piracicaba: FEALQ, 1999. 247 p.

LARRY, D.J.; STEVE, P.; WILLIAM, G.B. Troubleshooting swine ventilation systems. West Lafayette: Purdue University Cooperative Extensoin Service, 1994. 32 p. (PIH, 84)

MARTINS, L.C.; LATORRE, M.R.O.; CARDOSO, M.R.A.; GONÇALVES, F.L.T.; SALDIVA. P.H.N.; BRAGA, A.L.F. Poluição atmosférica e atendimentos por pneumonia e gripe em São Paulo, Brasil. Revista de Saúde Pública, São Paulo, v.36, n.1, p.88-94, 2002.

MÁSCIA, F.L.; SZNELWAR, L.I. Ergonomia: gestão de operações. 2.ed. São Paulo: Edgard Blucher, 2001. cap. 13, p.16-30.

MUTEL, C.F.; DONHAM, K.J. Occupational health problems of the rural work force. In: OSHA. Medical practices in rural communities. New York: Springer-Verlag, 1983. v.1, p.77-115.

NADER, A.; BARACHO, M.S.; NÄÄS, I.A.; SAMPAIO, C.A.P. Avaliação dos níveis de ruídos e da qualidade do ar (com relação à presença de gases e fungos) em creche de suínos. In SEMINÁRIO POLUENTES AÉRIOS E RUÍDOS EM INSTALAÇÕES PARA PRODUÇÃO DE ANIMAIS, 1., 2002, Campinas. Anais... Campinas: FEAGRI/UNICCAMP, 2002. p.49-56.

NI, J-Q.; HEBER, A.J.; DIEHL, C.A.; LIM, T.T.; DUGGIRALA, R.K.; HAYMORE, B.L. Hydrogen sulphide emission from two large pig-finishing buildings with long-term high-frequency measurements. Journal of Agricultural Science, Cambridge, v.138, n.2, p.227-236, 2002.

NIOSH. NATIONAL INSTITUTE FOR OCCUPATIONAL SAFETY AND HEALTH. Occupational health guidelines for carbon monoxide. Occupational health guidelines for chenical harzards, v.1, p.1-4, 1981.

NIOSH. NATIONAL INSTITUTE FOR OCCUPATIONAL SAFETY AND HEALTH. Manual of analytical methods (NMAM). $4^{\text {th }}$ ed. 1994. Disponível em: <WWw.cdc.gov/niosh/nmam/nmammenu.html>. Acesso em: 30 jan. 2007.

NORMA REGULAMENTADORA - NR-15. Ministério do Trabalho e Emprego. Portaria 3.214 de jul. 1978. Normas Regulamentadoras de segurança e saúde no trabalho (NR-15): atividades e operações insalubres. Brasília, $1978 . \quad$ Disponível em: $<$ http://www.mte.gov.br/temas/segsau/legislacao/normas/conteudo/nr15>. Acesso em: 20 nov. 2007. 
OSHA. OCCUPATIONAL SAFETY AND HEALTH ADMINISTRATION. Particulates not otherwise regulated, respirable 0600. In: NIOSH manual of analytical methods. $4^{\text {th }}$ ed. Disponível em: <www.cdc.gov/niosh/nmam/nmammenu.html>. Acesso em: 25 jan. 2006.

PERDOMO, C.C.; LIMA, G.J.M.M.; NONES, K. Produção de suínos e meio ambiente. In: SEMINÁRIO NACIONAL DE DESENVOLVIMENTO DA SUINOCULTURA, 90., 2001. Gramado. Anais... p.11-17. Disponível em: <http://www.cnpsa.embrapa.br/ > Acesso em: 10 dez. 2006.

PICKRELL, J. Hazards in confinement housing - gases and dusts in confined animal houses for swine, poultry, horses and humans. Veterinarian Human Toxicology, Manhattan, v.33, n.1, p.32-39, 1991.

PIFFER, I.A.; PERDOMO, C.C.; SOBESTIANSSKY, J. Efeitos de fatores ambientais na ocorrência de doenças. In: SOBESTIANSSKY, J.; WENTZ, I.; SESTI, L.C. Suinocultura Intensiva. Concórdia: Embrapa, 1998. 261 p.

SALIBA, T.M. Insalubridade e periculosidade: aspectos técnicas e práticas. 3.ed. São Paulo: LRT, 1997. $230 \mathrm{p}$.

SAMPAIO, C.S.; NÄÄS, I.A.; BARACHO, M.S.; SALGADO, D.D. Avaliação de poluentes aéreos em instalações de creche e terminação de suínos. Ciência Rural, Santa Maria, v.37, n.2, p.488-494, 2007.

SAMPAIO, C.S. Caracterização dos ambientes térmico, aéreo e acústico em sistemas de produção de suínos, nas fases de creche e terminação. 2004. $130 \mathrm{f}$. Tese (Doutorado em Construções Rurais e Ambiência) - Departamento de Construções Rurais e Ambiência, Universidade Estadual de Campinas, Campinas, 2004.

VERSTEGEN, M.; TAMMINGA, S.; GREERS, R. The effect of gaseous pollutants on animals. In: DEWI, I.A. Pollution in livestock production systems. Amsterdam: CAB International, 1994. 463 p. 